Check for updates

Cite this: Chem. Sci., 2019, 10, 6210

๑ All publication charges for this article have been paid for by the Royal Society of Chemistry

Received 26th April 2019 Accepted 15th May 2019

DOI: $10.1039 /$ c9sc02067e

rsc.li/chemical-science

\section{Catalytic protodeboronation of pinacol boronic esters: formal anti-Markovnikov hydromethylation of alkenes $\dagger$}

\author{
Florian Clausen, Marvin Kischkewitz, Klaus Bergander and Armido Studer (DD *
}

Pinacol boronic esters are highly valuable building blocks in organic synthesis. In contrast to the many protocols available on the functionalizing deboronation of alkyl boronic esters, protodeboronation is not well developed. Herein we report catalytic protodeboronation of $1^{\circ}, 2^{\circ}$ and $3^{\circ}$ alkyl boronic esters utilizing a radical approach. Paired with a Matteson- $\mathrm{CH}_{2}$-homologation, our protocol allows for formal anti-Markovnikov alkene hydromethylation, a valuable but unknown transformation. The hydromethylation sequence was applied to methoxy protected (-)- $\Delta 8-\mathrm{THC}$ and cholesterol. The protodeboronation was further used in the formal total synthesis of $\delta$ - $(R)$-coniceine and indolizidine $209 \mathrm{~B}$.
Organoboron compounds are highly valuable building blocks in organic synthesis. ${ }^{1,2}$ The most important application to be named is the Suzuki-Miyaura-coupling: ${ }^{3-5}$ Moreover, the boron moiety can be converted into a broad range of functional groups. $^{2,6}$ These transformations include oxidations, ${ }^{7}$ aminations, ${ }^{8-10}$ halogenations, ${ }^{11,12}$ and C-C-bond-formations such as alkenylations, ${ }^{13,14}$ alkynylations ${ }^{15}$ and arylations ${ }^{16}$ To access the B-building blocks, various borylation approaches have been developed over the years ${ }^{17-19}$ including the prominent asymmetric hydroboration reaction reported by H. C. Brown in $1961 .^{20}$

However, considering synthetic applications, drawbacks associated with organoboranes are their limited air and moisture stability. ${ }^{21}$ Along these lines, the introduction of the more stable boronic ester moiety has significantly expanded the scope of boron chemistry. In particular the pinacol boronic esters, which are usually bench stable,$^{22}$ easy to purify and often even commercially available have played a prominent role. These features are attractive for chemical transformations, where the valuable boron moiety remains in the product (homologations, ${ }^{23-25}$ conjunctive cross couplings ${ }^{26}$ or radical-polar crossover reactions $\mathrm{s}^{27-32}$ ). However, the increased stability also rises new challenges, considering the removal of the boron moiety at the end of a sequence if required. While boranes undergo efficient protodeboronation with propionic acid by protonolysis $^{33-35}$ or hydrogenation at elevated temperatures, ${ }^{36,37}$ boronic esters usually do not. ${ }^{21,22}$ We further noted that the protodeboronation of unactivated alkyl and especially primary alkyl boronic esters is underexplored. Reduction of $3^{\circ}$ alkyl

Organisch-Chemisches Institut, Westfälische Wilhelms-Universität, Corrensstraße 40, 48149 Münster, Germany. E-mail: studer@uni-muenster.de

$\dagger$ Electronic supplementary information (ESI) available. See DOI: 10.1039/c9sc02067e boronic esters was recently investigated by Aggarwal and coworkers; however, their method is only applicable to the protodeboronation of activated benzylic substrates. ${ }^{38}$ In 2005, Renaud and coworkers described an efficient sequence for the formal hydrogenation of unactivated alkenes to alkanes using a hydroboration-deboronation strategy (Scheme 1a). ${ }^{39}$ The protodeboronation of in situ generated catechol boronic esters proceeds via a radical chain reaction, but this reaction is limited to the more expensive catechol boronic esters and works well for $2^{\circ}$ alkyl B-esters. ${ }^{40}$

An elegant C-C-coupling/protodeboronation strategy was introduced in 2014 by Aggarwal and coworkers (Scheme 1b). ${ }^{41}$ In this sequence, the protodeboronation of $2^{\circ}$ alkyl pinacol boronic esters was achieved by oxidation of their corresponding methyl boron ate complexes with a stoichiometric amount of

a) Alkene hydrogenation via hydroboration-protodeboronation (Renaud 2005)

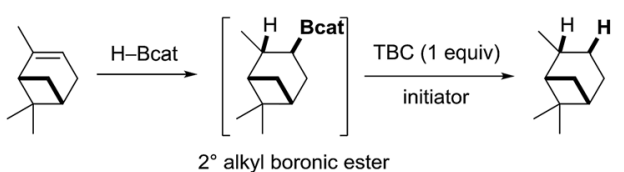

b) Alkyl carbamate/alkyl boronic ester coupling via protodeboronation (Aggarwal 2014)

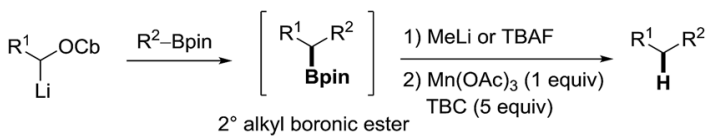

c) Alkene hydromethylation via hydroboration-homologation-protodeboronation (this work)

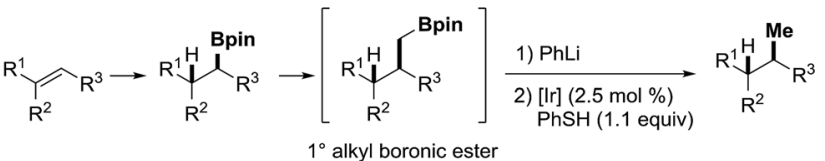

Scheme 1 Protodeboronation and its application in synthesis. 
manganese(III)acetate. The resulting secondary alkyl radicals are then reduced with 4-tert-butylcatechol (TBC). In contrast to the Renaud process, the latter protodeboronation works with pinacol boronic esters but does not proceed on primary B-esters (see below) and a large amount of reductant is required.

During a metabolite study, we targeted an anti-Markovnikovtype hydromethylation of a trisubstituted alkene and noted that different alkene hydromethylation approaches have been published over the years. ${ }^{42}$ However, all protocols developed so far provide the Markovnikov product. We therefore envisioned a hydroboration/ $/ \mathrm{CH}_{2}$-homologation/protodeboronation strategy to achieve anti-Markovnikov alkene hydromethylation (Scheme 1c). In this sequence, the regioselectivity is controlled in the initial hydroboration step, where suitable methods are available. ${ }^{43,44}$ The methylene unit can readily be introduced via established Matteson-homologation using $\mathrm{CH}_{2} \mathrm{Br}_{2}$ as the methylene source. The real challenge turned out to be the final protodeboronation of the intermediate $1^{\circ}$ alkyl boronic ester. The conditions reported by Aggarwal ${ }^{41}$ were ineffective for our systems and also other methods failed. We therefore initiated a project along those lines and aimed at a catalytic protodeboronation using boron ate complexes as substrates. Single electron oxidation by a redox catalyst should generate the corresponding alkyl radicals. ${ }^{45-47}$ Reduction of these alkyl radicals to give the targeted products should be achieved by thiols, since they are known to be efficient H-donors and the resulting thiyl radicals can be reduced by the photoredox catalyst thereby regenerating the initial oxidation state of the catalyst. ${ }^{48-51}$ Hence, an external stoichiometric oxidant is not required increasing the economy of the protodeboronation.

We commenced our investigations by testing different boron ate complexes derived from boronic ester 1a. In each case, the ate complex was generated in $\mathrm{Et}_{2} \mathrm{O}$. After removal of the ether, the residual ate complex was reacted with $2.5 \mathrm{~mol} \%$ of the Ircatalyst PC1 and thiophenol (1.1 equiv.) in $\mathrm{MeOH} /$ acetone at room temperature under blue light irradiation. B-ate complexes derived from TBAF, ${ }^{47} \mathrm{DMAP},{ }^{47} \mathrm{PPh}_{3}$ (ref. 47 ) and 3-quinolidinol $^{47}$ (1.1 equivalent each) did not afford the desired product 2a. However, with $\mathrm{PhLi}$, the target $2 \mathrm{a}$ was obtained in $79 \%$ yield (Scheme 2). Phenyl boronic ester 3 was formed as the stoichiometric byproduct. Notably, in a proof of principle experiment we demonstrated that the cascade also works efficiently by using catalytic amounts of the thiol $(10 \mathrm{~mol} \%)$ in combination with diphenyl phosphate (1.1 equiv.) as the external proton source (95\%, see ESI $\dagger$ ). However, by using $50 \mathrm{~mol} \%$ thiophenol in $\mathrm{MeOH} /$ acetone, the yield dropped significantly, showing the necessity of the phosphate in the thiol catalytic procedure. Since thiophenol is cheap, we decided to use the stoichiometric thiol protocol for the scope studies.

Under optimized conditions, various primary alkyl boronic esters 1b-n were tested (Scheme 2). Reactions proceeded smoothly and the products $\mathbf{2} \mathbf{b}-\mathbf{n}$ were isolated in moderate to excellent yields (52-93\%). Electron-rich arenes are well tolerated, as documented by the successful preparation of $\mathbf{2 b - c}, 2 \mathbf{g}$ and 2k. Furthermore, substrates bearing a triphenyl silane (1i) and a carbazole moiety (1j) performed well and the desired products $2 \mathbf{i}-\mathbf{j}$ were isolated in $72 \%$ and $88 \%$ yield, respectively.

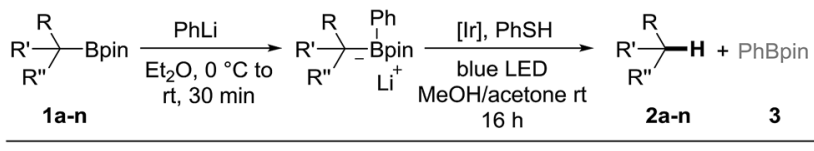

$1^{\circ}$
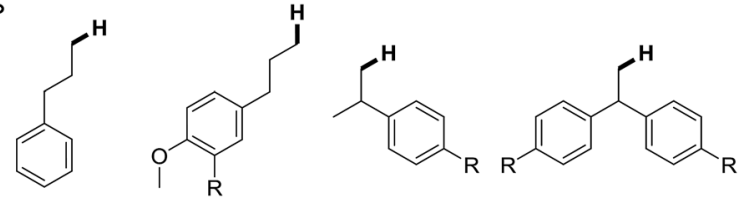

$2 \mathrm{a}(79 \%)^{\mathrm{a}}$

$\begin{array}{ll}\text { 2b R }=H(63 \%) & \text { 2d } \mathrm{R}=\mathrm{Ph}(86 \%) \\ \text { 2c } \mathrm{R}=\mathrm{OMe}(92 \%) & \text { 2e } \mathrm{R}=t-\mathrm{Bu}(76 \%)\end{array}$

2f $\mathrm{R}=\mathrm{H}(75 \%)$

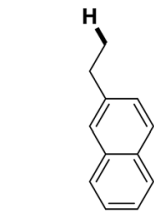

2h (64\%) $(61 \%$, gram scale)

$2^{\circ}$

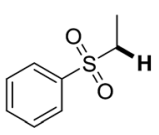

2I (85\%)

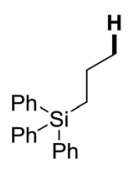

$2 \mathbf{i}(72 \%)$

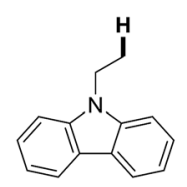

2j (88\%)

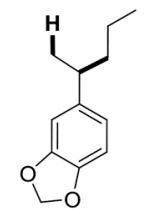

2k (53\%, 92\% ee)

$-$




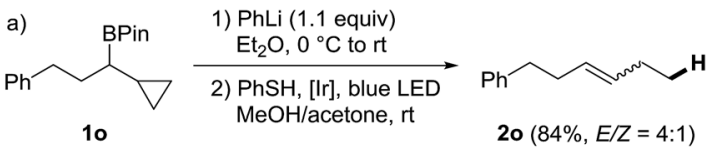

b)

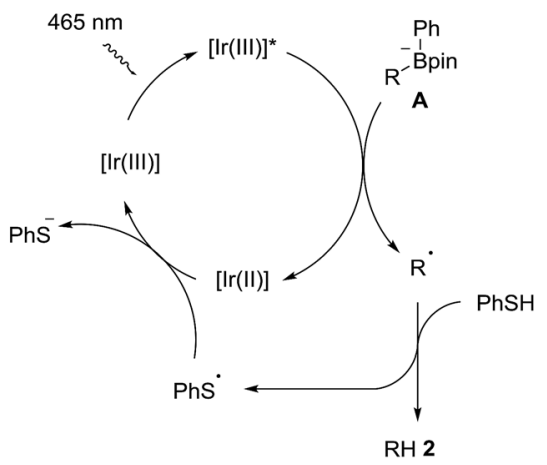

Scheme 3 Radical probe experiment and proposed mechanism.

thiyl radical, which is reduced by the Ir(II)-complex to regenerate the starting Ir(III)-complex PC1.

To further document the potential of the protodeboronation, we applied the novel protocol to the formal total synthesis of two indolizidine-core containing secondary metabolites, $\delta-(R)$ coniceine (9) and indolizidine 209B (13) (Scheme 4). Starting with commercial $N$-Boc-pyrrolidine (4), enantiopure boronic ester 5 was prepared via a (+)-sparteine mediated asymmetric lithiation/borylation..$^{54-56}$ The ester moiety was introduced via stereospecific radical-induced 1,2-migration ${ }^{27,28}$ of the corresponding in situ generated vinyl and isopropenyl boron ate complexes with $\alpha$-iodo acetates as radical precursors to provide 6 and 10 in $52 \%$ and $65 \%$ yield. In the coniceine route, 6 was directly subjected to the protodeboronation to provide the pyrrolidine 7 in $61 \%$ yield. After ester cleavage and Bocdeprotection, the corresponding carboxylic acid was cyclised with EDC and DMAP to yield the bicyclic lactam 8 in $87 \%$ yield, completing the formal total synthesis ${ }^{57}$ of $\delta$ - $(R)$-coniceine (9). A crucial step in the indolizidine 209B synthesis was the build-up of the additional C-8 stereocenter via a stereoselective protodeboronation. To this end, the $3^{\circ}$ boronic ester 10 was converted into the rigid indolizidine precursor 11 via Bocdeprotection and lactamization (68\%). Protodeboronation by using the less nucleophilic (3,5-bis(trifluoromethyl)phenyl) lithium for boron ate complex formation in place of $\mathrm{PhLi}$, to prevent aryl addition to the lactam moiety, afforded the indolizidine 12 in 59\% yield and good diastereoselectivity ( $\mathrm{dr}=$ 5 : 1). Final transformation into indolizidine 209B (13) can be achieved by Grignard-addition to the lactam followed by stereoselective reductive deoxygenation. ${ }^{58}$

Finally, we focused on the formal alkene hydromethylation sequence (Scheme 5). Initial studies were conducted with the boronic esters 14a-d which can be obtained via hydroboration of the corresponding styrene derivatives (see the ESI $\dagger$ ). The Matteson- $\mathrm{CH}_{2}$-homologation was carried out with in situ generated $\mathrm{CH}_{2} \mathrm{BrLi}$ in THF at low temperature. After removal of the solvent, the crude homologated boronic esters 15ad were directly subjected to the protodeboronation to provide the targeted compounds 16a-d in $65-66 \%$ overall yields. The heteroarenes 14e, 14f and a silylated boronic ester were eligible substrates and provided $16 \mathbf{e}-\mathbf{g}$ in $51-70 \%$ yield. When the OTs-protected phenol $\mathbf{1 4 h}$ was subjected to the sequence, the deprotected phenol $\mathbf{1 6 h}$ resulted (48\%). The sequence was also applied to the hydromethylation of more complex alkenes such as the fluorine containing system 14d to obtain the hydromethylated compound $\mathbf{1 6 d}$ in $66 \%$ yield. The hetero arenes $14 \mathrm{e}$ and $\mathbf{1 4 f}$ were tolerated and provided the products 16e and 16f in 51-55\% and yields. When the OTs-protected phenol 14h was subjected to the sequence the deprotected compound $16 \mathrm{~h}$ was isolated in $48 \%$ yield. The method was also applied to cyclic olefins such as the cyclohexane derivative $14 \mathbf{i}$ to give the product $16 \mathbf{i}$ in $61 \%$ yield, the methyl protected $(-)-\Delta^{8}$-THC $\mathbf{1 4} \mathbf{j}$ and the cholesterol derivative $\mathbf{1 4 k}$. Stereo- and regioselective hydroboration using catecholborane under neat conditions ${ }^{\mathbf{1 6}}$ followed by transesterification with pinacol gave the $2^{\circ}$ alkyl boronic esters $14 \mathbf{j}$ and $14 \mathbf{k}$ in $55 \%$ to $65 \%$ yield as single diastereoisomers (see ESI $\dagger$ ). $\mathrm{CH}_{2}$ -

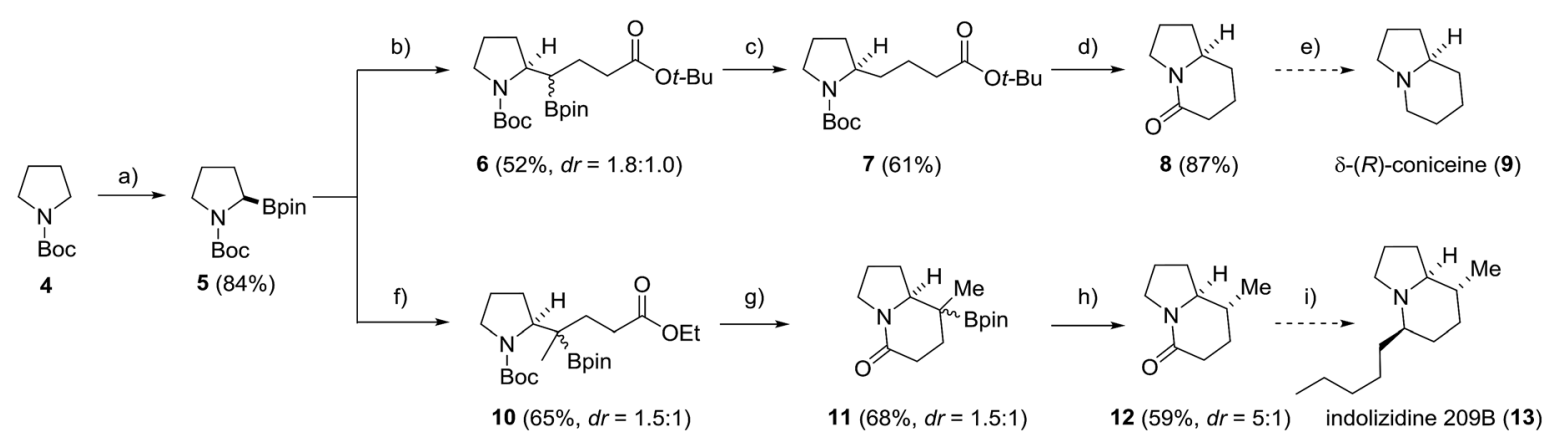

Scheme 4 Formal total synthesis of $\delta$-(R)-coniceine (9) and indolizidine 209B (13). Reagents and conditions: (a) s-BuLi (1.2 equiv.), (+)-sparteine (1.2 equiv.) $\mathrm{Et}_{2} \mathrm{O},-78^{\circ} \mathrm{C}, 3 \mathrm{~h}$ then isopropyl pinacol borate $\left(1.3\right.$ equiv.), $-78^{\circ} \mathrm{C}, 2 \mathrm{~h}$; (b) vinyllithium (1.3 equiv.), $\mathrm{Et}_{2} \mathrm{O},-78^{\circ} \mathrm{C}$ to rt, $30 \mathrm{~min}$ then tertbutyl 2-iodoacetate (2.0 equiv.), $\mathrm{MeCN}, h \nu, 50{ }^{\circ} \mathrm{C}, 24 \mathrm{~h}$; (c) PhLi (1.1 equiv.), $\mathrm{Et}_{2} \mathrm{O}, 0{ }^{\circ} \mathrm{C}$ to rt, 30 min then PhSH (1.1 equiv.), PC1 (2.5\%), blue LED, $\mathrm{MeOH} /$ acetone, $16 \mathrm{~h}$; (d) $\mathrm{HCl}$ in dioxane, $\mathrm{rt}, 8 \mathrm{~h}$ then $\mathrm{EDC} \cdot \mathrm{HCl}\left(2.0\right.$ equiv.), DMAP (20 mol\%), Hünig's base $\left(2.0\right.$ equiv.), $\mathrm{CH}_{2} \mathrm{Cl}_{2}, \mathrm{rt}$, over night; (e) see ref. 57 ; (f) isopropenyllithium (1.3 equiv.), $\mathrm{Et}_{2} \mathrm{O},-78^{\circ} \mathrm{C}$ to rt, $30 \mathrm{~min}$ then ethyl 2 -iodoacetate $\left(5.0\right.$ equiv.), $\mathrm{MeCN}, h \nu, 50{ }^{\circ} \mathrm{C}, 24 \mathrm{~h}$; (g) TFA (10 eq.), $\mathrm{CH}_{2} \mathrm{Cl}_{2}, 0{ }^{\circ} \mathrm{C}$ to $\mathrm{rt}, 2 \mathrm{~h}$ then $\mathrm{NEt}_{3}\left(20\right.$ equiv.), $\mathrm{CH}_{2} \mathrm{Cl}_{2}, \mathrm{rt}, 12 \mathrm{~h}$; (h) (3,5-bis(trifluoromethyl)phenyl)lithium (1.3 equiv.), $\mathrm{Et}_{2} \mathrm{O},-78{ }^{\circ} \mathrm{C}, 1 \mathrm{~h}$ then PhSH (1.3 equiv.), PC1 (2.5\%), blue LED, MeOH/acetone, 16 h; (i) see ref. 58. 

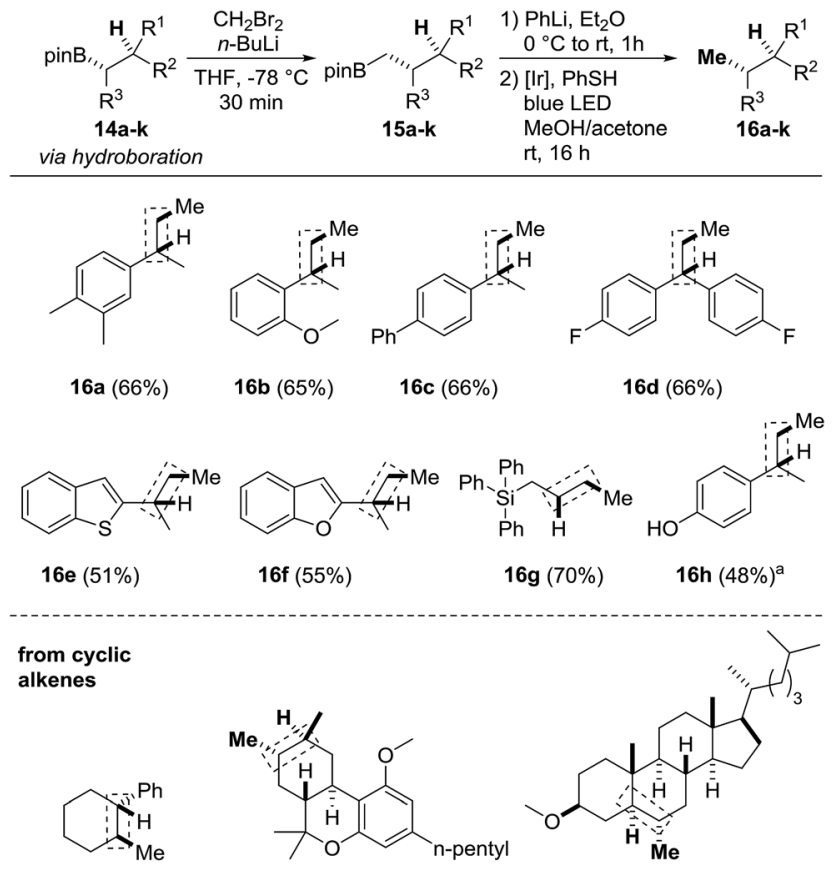

16b $(65 \%)$

16c $(66 \%)$

16d (66\%)
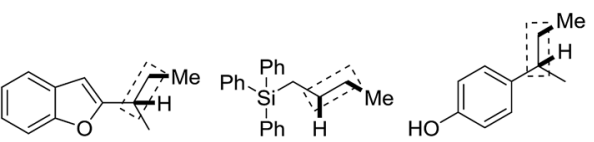

16i (61\%)
$16 f(55 \%)$

16g $(70 \%)$

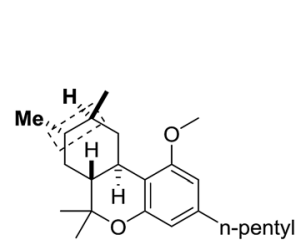

16j $(90 \%)$

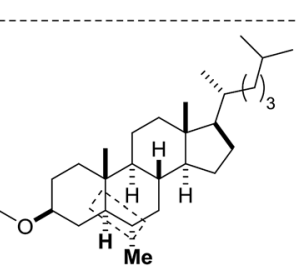

16k (89\%)
Scheme 5 Formal alkene hydromethylation. Homologation: $14 \mathrm{a}-\mathrm{k}$ (0.05-0.2 mmol, 1.0 equiv.), $\mathrm{CH}_{2} \mathrm{Br}_{2}$ (2.0-3.0 equiv.), $n$-butyllithium (1.5-2.0 equiv.) in $\mathrm{THF}$ or $\mathrm{Et}_{2} \mathrm{O}$ : protodeboronation: $15 \mathrm{a}-\mathrm{k}(0.05-$ $0.2 \mathrm{mmol}, 1.0$ equiv., used as crude from homologation step), PhLi (1.1 equiv.), $\mathrm{Et}_{2} \mathrm{O}(0.5-2.0 \mathrm{~mL})$, thiophenol (1.1 equiv.), PC1 (2.5 mol\%) in $\mathrm{MeOH} /$ acetone $(1: 1,0.5-2.0 \mathrm{~mL})$. Yield corresponds to the two-step homologation-protodeboronation sequence. For the hydroboration step, we refer to the ESI. $\dagger^{\mathrm{a}}$ Derived from O-Ts-protected phenol, O-S cleavage during reaction.

homologation and protodeboronation afforded the methylated products $\mathbf{1 6 \mathbf { j }}$ and $\mathbf{1 6 k}$ in excellent overall yields (89\% and $90 \%)$, convincingly documenting the potential of our novel sequence.

In summary, we have developed the first catalytic protodeboronation of unactivated $1^{\circ}, 2^{\circ}$ and $3^{\circ}$ alkyl pinacol boronic esters utilizing photoredox catalysis. We were able to carry out a formal methane addition to various alkenes combining the protodeboronation protocol with a Matteson- $\mathrm{CH}_{2}$-homologation. The sequence was applied to the hydromethylation of secondary metabolite derivatives, a $(-)-\Delta^{8}$-THC derivative and $O$-methylated cholesterol. Furthermore, the protodeboronation was successfully also used in formal total syntheses of $\delta-(R)$ coniceine (9) and indolizidine 209B (13).

\section{Conflicts of interest}

The authors declare no conflict of interest.

\section{Acknowledgements}

We thank the European Research Council (ERC Advanced Grant agreement no. 692640) for financial support.

\section{Notes and references}

1 Synthesis and Application of Organoboron Compounds, ed. E. Fernández and A. Whiting, Springer International Publishing, 2015.

2 C. Sandford and V. K. Aggarwal, Chem. Commun., 2017, 53, 5481-5494.

3 N. Miyaura, K. Yamada and A. Suzuki, Tetrahedron Lett., 1979, 20, 3437-3440.

4 N. Miyaura and A. Suzuki, Chem. Rev., 1995, 95, 2457-2483.

5 A. Suzuki, Angew. Chem., Int. Ed., 2011, 50, 6722-6737.

6 H. K. Scott and V. K. Aggarwal, Chem.-Eur. J., 2011, 17, 13124-13132.

7 H. C. Brown and B. C. S. Rao, J. Am. Chem. Soc., 1956, 78, 5694-5695.

8 H. C. Brown, M. M. Midland and A. B. Levy, J. Am. Chem. Soc., 1973, 95, 2394-2396.

9 H. C. Brown, A. M. Salunkhe and A. B. Argade, Organometallics, 1992, 11, 3094-3097.

10 S. N. Mlynarski, A. S. Karns and J. P. Morken, J. Am. Chem. Soc., 2012, 134, 16449-16451.

11 H. C. Brown and C. F. Lane, J. Chem. Soc., Chem. Commun., 1971, 521-522.

12 H. C. Brown, N. R. De Lue, G. W. Kabalka and H. C. Hedgecock, J. Am. Chem. Soc., 1976, 98, 1290-1291.

13 R. J. Armstrong and V. K. Aggarwal, Synthesis, 2017, 49, 33233336.

14 G. Zweifel, H. Arzoumanian and C. C. Whitney, J. Am. Chem. Soc., 1967, 89, 3652-3653.

15 Y. Wang, A. Noble, E. L. Myers and V. K. Aggarwal, Angew. Chem., Int. Ed., 2016, 55, 4270-4274; Y. Wang, A. Noble, E. L. Myers and V. K. Aggarwal, Angew. Chem., 2016, 128, 4342.

16 A. Bonet, M. Odachowski, D. Leonori, S. Essafi and V. K. Aggarwal, Nat. Chem., 2014, 6, 584-589.

17 Y. Cheng, C. Mück-Lichtenfeld and A. Studer, Angew. Chem., Int. Ed., 2018, 57, 16832-16836; Y. Cheng, C. MückLichtenfeld and A. Studer, Angew. Chem., 2018, 130, 17074.

18 A. Fawcett, J. Pradeilles, Y. Wang, T. Mutsuga, E. L. Myers and V. K. Aggarwal, Science, 2017, 357, 283-286.

19 C. Li, J. Wang, L. M. Barton, S. Yu, M. Tian, D. S. Peters, M. Kumar, A. W. Yu, K. A. Johnson, A. K. Chatterjee, et al., Science, 2017, 356, 7355.

20 H. C. Brown and G. Zweifel, J. Am. Chem. Soc., 1961, 83, 486487.

21 D. S. Matteson, J. Organomet. Chem., 1999, 581, 51-65.

22 R. Bernardini, A. Oliva, A. Paganelli, E. Menta, M. Grugni, S. D. Munari and L. Goldoni, Chem. Lett., 2009, 38, 750-751.

23 M. Burns, S. Essafi, J. R. Bame, S. P. Bull, M. P. Webster, S. Balieu, J. W. Dale, C. P. Butts, J. N. Harvey and V. K. Aggarwal, Nature, 2014, 513, 183-188.

24 J. Gorges and U. Kazmaier, Org. Lett., 2018, 20, 2033-2036. 25 D. S. Matteson, J. Org. Chem., 2013, 78, 10009-10023.

26 L. Zhang, G. J. Lovinger, E. K. Edelstein, A. A. Szymaniak, M. P. Chierchia and J. P. Morken, Science, 2016, 351, 7074. 
27 M. Kischkewitz, K. Okamoto, C. Mück-Lichtenfeld and A. Studer, Science, 2017, 355, 936-938.

28 C. Gerleve, M. Kischkewitz and A. Studer, Angew. Chem., Int. Ed., 2018, 57, 2441-2444; C. Gerleve, M. Kischkewitz and A. Studer, Angew. Chem., 2018, 130, 2466.

29 A. Noble, R. S. Mega, D. Pflästerer, E. L. Myers and V. K. Aggarwal, Angew. Chem., Int. Ed., 2018, 57, 2155-2159.

30 N. D. C. Tappin, M. Gnägi-Lux and P. Renaud, Chem.-Eur. J., 2018, 24, 11498-11502.

31 M. Kischkewitz, Org. Synth., 2018, 95, 205-217.

32 M. Kischkewitz, C. Gerleve and A. Studer, Org. Lett., 2018, 20, 3666-3669.

33 H. C. Brown and K. Murray, J. Org. Chem., 1961, 26, 631-632.

34 H. C. Brown and K. Murray, J. Am. Chem. Soc., 1959, 81, 41084109.

35 H. C. Brown and K. J. Murray, Tetrahedron, 1986, 42, 54975504.

36 R. Köster, Angew. Chem., 1956, 68, 383.

37 F. L. Ramp, E. J. DeWitt and L. E. Trapasso, J. Org. Chem., 1962, 27, 4368-4372.

38 S. Nave, R. P. Sonawane, T. G. Elford and V. K. Aggarwal, J. Am. Chem. Soc., 2010, 132, 17096-17098.

39 D. Pozzi, E. M. Scanlan and P. Renaud, J. Am. Chem. Soc., 2005, 127, 14204-14205.

40 G. Villa, G. Povie and P. Renaud, J. Am. Chem. Soc., 2011, 133, 5913-5920.

41 R. Rasappan and V. K. Aggarwal, Nat. Chem., 2014, 6, 810814.

42 H. T. Dao, C. Li, Q. Michaudel, B. D. Maxwell and P. S. Baran, J. Am. Chem. Soc., 2015, 137, 8046-8049.

43 M. Odachowski, A. Bonet, S. Essafi, P. Conti-Ramsden, J. N. Harvey, D. Leonori and V. K. Aggarwal, J. Am. Chem. Soc., 2016, 138, 9521-9532.

44 R. Soundararajan and D. S. Matteson, J. Org. Chem., 1990, 55(8), 2274-2275.
45 G. Duret, R. Quinlan, P. Bisseret and N. Blanchard, Chem. Sci., 2015, 6, 5366-5382.

46 C. Shu, A. Noble and V. K. Aggarwal, Angew. Chem., Int. Ed., 2019, 58, 3870; C. Shu, A. Noble and V. K. Aggarwal, Angew. Chem., 2019, 131, 3910.

47 F. Lima, M. A. Kabeshov, D. N. Tran, C. Battilocchio, J. Sedelmeier, G. Sedelmeier, B. Schenkel and S. V. Ley, Angew. Chem., Int. Ed., 2016, 55, 14085; F. Lima, M. A. Kabeshov, D. N. Tran, C. Battilocchio, J. Sedelmeier, G. Sedelmeier, B. Schenkel and S. V. Ley, Angew. Chem., 2016, 128, 14291.

48 Q. Zhu, D. E. Graff and R. R. Knowles, J. Am. Chem. Soc., 2018, 140, 741-747.

49 A. J. Musacchio, B. C. Lainhart, X. Zhang, S. G. Naguib, T. C. Sherwood and R. R. Knowles, Science, 2017, 355, 727730.

$50 \mathrm{H}$. Jiang and A. Studer, Chem.-Eur. J., DOI: 10.1002/ chem.201901566.

51 J. D. Griffin, M. A. Zeller and D. A. Nicewicz, J. Am. Chem. Soc., 1993, 115, 939-947.

52 J. Chen, T. Xi, X. Ren, B. Cheng, J. Guo and Z. Lu, Org. Chem. Front., 2014, 1, 1306-1309.

53 K. E. Liu, C. C. Johnson, M. Newcomb and S. J. Lippard, J. Am. Chem. Soc., 1993, 115, 939-947.

54 A. Varela, L. K. B. Garve, D. Leonori and V. K. Aggarwal, Angew. Chem., Int. Ed., 2017, 56, 2127-2131; A. Varela, L. K. B. Garve, D. Leonori and V. K. Aggarwal, Angew. Chem., 2017, 129, 2159.

55 D. Hoppe and T. Hense, Angew. Chem., Int. Ed. Engl., 1997, 36, 2282-2316.

56 S. Wu, S. Lee and P. Beak, J. Am. Chem. Soc., 1996, 118, 715721.

57 S. H. Park, H. J. Kang, S. Ko, S. Park and S. Chang, Tetrahedron: Asymmetry, 2001, 12, 2621-2624.

58 H. Wu, M. Yu, Y. Zhang and G. Zhao, Chin. J. Chem., 2009, 27, 183-188. 